\title{
Design, Implementation, and Validation of Robust Fractional-Order PD Controller for Wheeled Mobile Robot Trajectory Tracking
}

\author{
Lichuan Zhang $\mathbb{D}^{1},{ }^{1}$ Lu Liu $\mathbb{D}^{1},{ }^{1}$ and Shuo Zhang $\mathbb{B}^{2}$ \\ ${ }^{1}$ School of Marine Science and Technology, Northwestern Polytechnical University, Xi'an 710072, China \\ ${ }^{2}$ Department of Applied Mathematics, Northwestern Polytechnical University, Xi'an 710072, China \\ Correspondence should be addressed to Lu Liu; liulu12201220@nwpu.edu.cn
}

Received 28 August 2019; Accepted 26 November 2019; Published 1 February 2020

Guest Editor: Raúl Villafuerte-Segura

Copyright ( 92020 Lichuan Zhang et al. This is an open access article distributed under the Creative Commons Attribution License, which permits unrestricted use, distribution, and reproduction in any medium, provided the original work is properly cited.

In this paper, a trajectory tracking control algorithm is proposed based on the fractional-order PD (FOPD) controller for a Wheeled Mobile Robot (WMR). Firstly, an improved flat phase property is put forward as a robust controller tuning specification. This specification is capable of guaranteeing the flatness of the phase curve in a frequency interval, so the controlled system robustness can be improved. Then, the stabilization process is discussed with respect to the parameters of the FOPD controller through a visualized 3-dimensional surface, so both the stability and robustness of the controlled system can be guaranteed under the proposed controller. Furthermore, the implementation of the proposed robust FOPD controller is presented, which makes the control algorithm easy to be realized. At last, the effectiveness of the proposed trajectory tracking control algorithm is verified by the simulation and experiment results.

\section{Introduction}

Wheeled Mobile Robots (WMRs) are capable of working in different situations, including the inclement, dangerous, or even harmful ones. In the past few decades, WMRs have been widely applied in a great variety of civilian and military tasks, for example, space exploration, materials transportation, supplies delivery, mine clearance, and search and rescue [1-6]. Currently, trajectory tracking and regulation is one of the most concerned problems in WMRs related studies [7]. Without an effective control strategy, a predefined tracking strategy is hard to follow, especially in long distance or complicated environment tasks. The nonholomic properties, internal dynamics, feedback sensors of WMRs, and external load disturbance may bring in different kinds of immeasurable uncertainties [8]. Therefore, more precise and robust trajectory tracking strategy will certainly help in improving the operation efficiency of WMRs.

Typically, Proportional-Integral-Derivative (PID) controller is always used in the control process of industrial robotics including WMRs. But its control effect has been suspicious at times when better robustness and transient performance are required. However, the combination of fractional calculus and traditional PID controller, namely, fractional-order PID (FOPID) controller, provides novel potential and opportunity of solving this kind of problem. Many studies have investigated the design and application of FOPID type controllers [9-11]. One of the most representative works is the $\mathrm{PI}^{\lambda} D^{\mu}$ controller proposed by Podlubny, which is an extension of the traditional PID controller with two extra order parameters [10]. Another kind of FOPID controller designed based on phase and magnitude margin frequency specifications is presented by Vinagre et al. [12]. Except for phase and magnitude margin specifications, Monje et.al. added the extrasensitivity and complementary sensitivity functions in the design specifications of the FOPID controller [13]. A similar FOPI controller whose parameters were tuned by symmetrical optimization function was studied by Maione and Lino [14]. In [15, 16], the original and modified Ziegler-Nichols methods were used in 
the design process of FOPID controllers. The NewtonRaphson recursive algorithm was applied by Feliu et al. in the parameter optimization process of an FOPI controller [17]. Moreover, some intelligent control algorithm-based FOPID tuning methods have also been studied [18-22]. An autotuning variable-order fuzzy FOPID controller is investigated in [18] by Liu et al. in which all the parameters can be tuned online to deal with random time delay or system parameter uncertainties. The detailed advantages of the FOPID type controller can be concluded as following. Firstly, the FOPID type controller inherits the characteristics of the traditional PID controller, such as simple structure, clear physical, and meaning. Secondly, through the extradifferential and integral order parameters, the design flexibility of the controller is increased so better dynamic performance and robustness may be achieved with this type of controller. Subsequently, the FOPID type controller has the memory property and can adjust the output of the controller properly following the error history information in order to obtain better performance. At last, the added fractional-order terms can help adjust the high-frequency and low-frequency characteristics of the closed-loop easily [23].

In addition, the practical applicability of the FO controller has been verified in some practical applications. Several FO control examples, including industrial plants and electromechanic actuator, are presented in [24]. A brief summary and introduction of FOPID controllers applied in various kinds of industrial situations is given in [25]. The CRONE controller which can compensate the disturbance caused by parameter uncertainties and load changes has been successfully used in vehicle suspension [26] and path tracking [27]. A series of robust FOPID controllers tuned based on the flat phase property have been used in motion control [28, 29]. Moreover, in [30], an FO controller is used in the trajectory tracking task of a differential drive WMR. The experimental results show that the control performance of the FO controller is more satisfactory compared with the traditional PD controller. Another speed and direction control task accomplished by the FOPI controller for skid-steered WMR is presented in [31], which also achieves superior control performance. Nevertheless, the related studies are still quite limited at present. The stability of the controlled systems and the advantages of FO controllers applied in speed regulation, trajectory tracking, and other practical applications of WMRs need more exploration.

The main contribution of this paper includes the design, implementation, and validation of an FOPD controller for precise trajectory tracking of WMRs. For robust FO controller regulation, the flat phase property has been widely used as a design specification $[29,32]$. This is because it can guarantee the flatness of the phase curve around the interested crossover frequency $\omega_{c}$, so the system phase margin can remain constant and the system is robust to load variations. However, there are mainly two drawbacks of the original flat phase property. The first one is that it can only guarantee the flatness of the phase curve at one frequency, namely, $\omega_{c}$. The other one is there are several nonlinear equations to be solved in the controller design process, so it is hard to find a solution. The improved flat phase property proposed in this paper can guarantee the robust flat phase frequency interval width instead of only one flat phase frequency and at least one parameter set which satisfies all the specifications can be found. In addition, the system stabilization and implementation problems are also discussed. The simulation and experiments results are shown to verify the effectiveness of the proposed trajectory tracking control algorithm.

The rest of this paper is organized as follows: In Section 2, the modelling of XQ WMR is given; then, the fractional calculus and FOPD controller are presented in Section 3; Section 4 shows the proposed controller design specifications; Section 5 discusses the stabilizing process for FO delay systems with the FOPD controller; furthermore, the experiment results and discussion are illustrated in Section 6 to verify the effectiveness and flexibility of the proposed controller; finally, the conclusion is drawn in Section 7.

\section{Modelling of WMR}

The XQ WMR studied in this paper is a three-wheeled differential drive robot, as shown in Figure 1.

2.1. Kinematic Model of XQ WMR. There are two front drive wheels and a universal follower wheel on the chassis of XQ WMR. The two front wheels driven by DC motors provide the forward power, so the movement and orientation of XQ WMR can be accomplished by the velocity difference between the two drive wheels; the universal follower wheel is mounted on the rear of the chassis. The kinematic model of XQ WMR is illustrated in Figure 2. As it is shown in Figure 2, denote $v_{\mathrm{c}}, v_{\mathrm{r}}$, and $v_{\mathrm{l}}$ as the chassis linear, right wheel, and left wheel velocities, respectively, $\theta$ as the angular displacement and $\omega$ as the angular velocity, where $\dot{\theta}=\omega$, and $2 R$ as the distance between the two drive wheels. Denote the state vector $S$ of XQ WMR as follows:

$$
S=\left[\begin{array}{l}
x \\
y \\
\theta
\end{array}\right],
$$

where $x$ and $y$ are coordinates of the center mass of XQ WMR. Therefore, the forward kinematic model of XQ WMR can be obtained as follows:

$$
\dot{S}=\left[\begin{array}{c}
\dot{x} \\
\dot{y} \\
\dot{\theta}
\end{array}\right]=\left[\begin{array}{c}
v_{\mathrm{c}} \cos (\theta) \\
v_{\mathrm{c}} \sin (\theta) \\
\omega
\end{array}\right]=\left[\begin{array}{c}
\frac{1}{2}\left(v_{\mathrm{r}}+v_{\mathrm{l}}\right) \cos (\theta) \\
\frac{1}{2}\left(v_{\mathrm{r}}+v_{\mathrm{l}}\right) \sin (\theta) \\
\omega
\end{array}\right] .
$$

Then, in order to achieve the input desirable right and left wheel velocities, the inverse kinematic model of XQ WMR is achieved as 


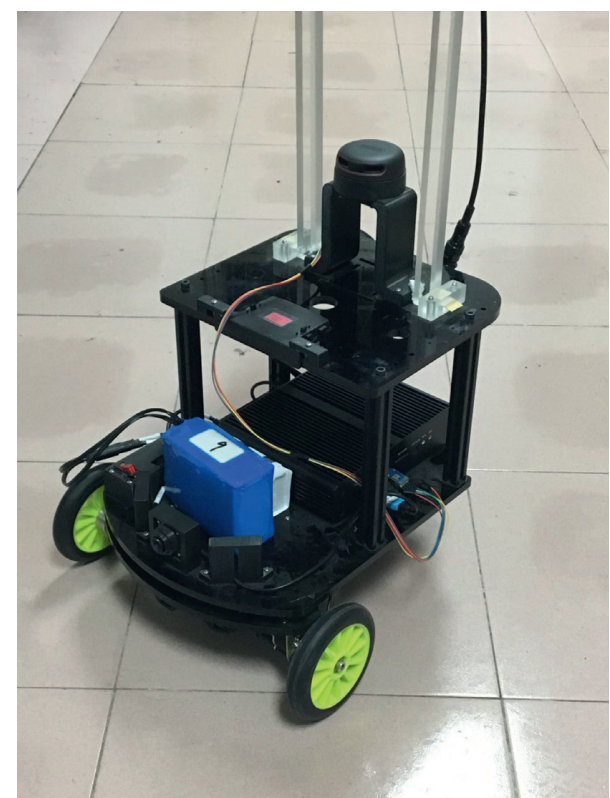

Figure 1: XQ WMR.

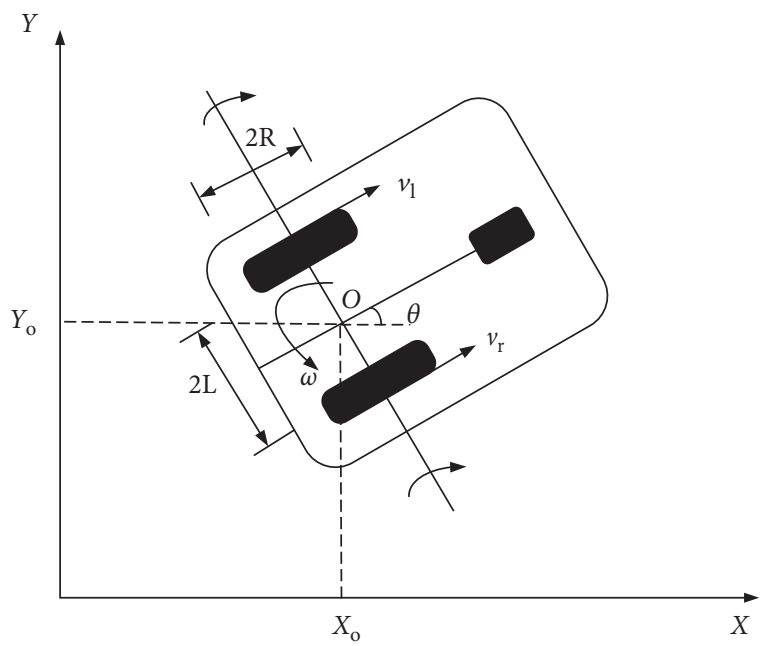

FIgURE 2: XQ WMR kinematic model.

$$
\left[\begin{array}{l}
v_{\mathrm{r}} \\
v_{\mathrm{l}}
\end{array}\right]=\left[\begin{array}{cc}
1 & R \\
1 & -R
\end{array}\right]\left[\begin{array}{l}
v_{\mathrm{c}} \\
\omega
\end{array}\right] .
$$

The control scheme proposed in this paper is shown in Figure 3. Note that the centre of the two drive wheels $O$ is assumed to be the mass centre of XQ WMR.

2.2. Motor Dynamic Model of XQ WMR. Before controller design procedure, the motor dynamic model should be achieved first. Figure 4 is the schematic diagram of the DC motor, where $R_{m}$ and $L_{m}$ are the total resistance and total inductance of the armature winding; $V_{m}$ is the armature voltage; $T(s)$ is the electromagnetic torque; $\omega_{m}$ is the rotational angular velocity of the motor; and $J_{m}$ is the rotational inertia on the deceleration shaft.
The transfer function of XQ WMR DC motor can be achieved as follows:

$$
G(s)=\frac{\theta(s)}{V_{m}(s)}=\frac{k_{m}}{s\left[\left(L_{m} s+R_{m}\right)\left(J_{m} s+k_{f}\right)+k_{b} k_{m}\right]},
$$

where $k_{f}$ and $k_{m}$ are constants related to the magnetic flux. Ignoring some of the negligible values and the parameters which have less impact on the overall mathematical model, a simplified DC mathematical model can be obtained as a second-order transfer function as follows:

$$
G(s)=\frac{\theta(s)}{V_{m}(s)}=\frac{k_{m}}{s\left[\left(L_{m} s+R_{m}\right)\left(J_{m} s+k_{f}\right)+k_{b} k_{m}\right]}=\frac{K}{s(\tau s+1)},
$$

where the time constant is $\tau=R_{m} J_{m} /\left(R_{m} k_{f}+k_{b} k_{m}\right)$ and $K=k_{m} /\left(R_{m} k_{f}+k_{b} k_{m}\right)$.

From the experimental identification, the parameters of the DC motor are achieved as $\tau=0.325$ and $K=7.74 / 20$.

\section{Fractional Calculus and FOPD Controller}

3.1. Fractional-Order Derivative. Fractional calculus, which is an extension of traditional calculus, has not got a unified definition so far. There are three definitions which have been extensively used [10, 33], namely, Grunwald-Letnikov definition, Riemann-Liouville definition, and Caputo definition. Each of the definitions has its own properties. The definitions should be applied appropriately in different research fields, such as engineering, applied mathematics, and computer science. The initial values of Caputo definition are the same with that of the integer-order system and own practical physical meaning $[34,35]$. Therefore, the Caputo definition is used in this paper.

An FO integral-differential operator ${ }_{t_{0}} D_{t}^{\delta}$ can be depicted as follows:

$$
t_{0} D_{t}^{\delta}= \begin{cases}\int_{t_{0}}^{t} f(\tau) \mathrm{d} \tau^{-\delta}, \delta<0, \\ f(t), & \delta=0, \\ \frac{\mathrm{d}^{\delta}}{\mathrm{d} t^{\delta}} f(t), & \delta>0,\end{cases}
$$

where $\delta \in R$ is the integral or differential order and $t_{0}$ and $t$ are lower and upper limits of the FO operator, respectively.

The Caputo derivative of order $\delta$ for a function $f(t) \in C^{m+1}\left(\left[t_{0},+\infty\right], R\right)$ is defined as follows[33]:

$$
{ }_{t_{0}} D_{t}^{\delta} f(t)=\frac{1}{\Gamma(m-\delta)} \int_{t_{0}}^{t} \frac{f^{(m)}(\tau)}{(t-\tau)^{\delta+1-m}} \mathrm{~d} \tau,
$$

where $m-1<\delta \leq m$, and $m$ is a positive integer.

The Laplace transformation corresponding to Caputo definition can be obtained as follows:

$$
\mathscr{L}\left\{{ }_{t_{0}} D_{t}^{\delta} f(t)\right\}=s^{\delta} F(s)-\sum_{k=0}^{m-1} s^{\delta-k-1} f^{(k)}\left(t_{0}\right),
$$




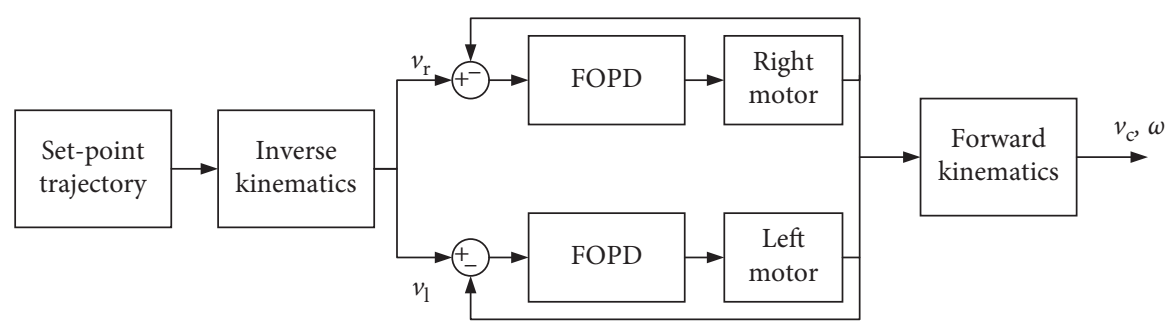

FIGURE 3: XQ WMR control strategy.

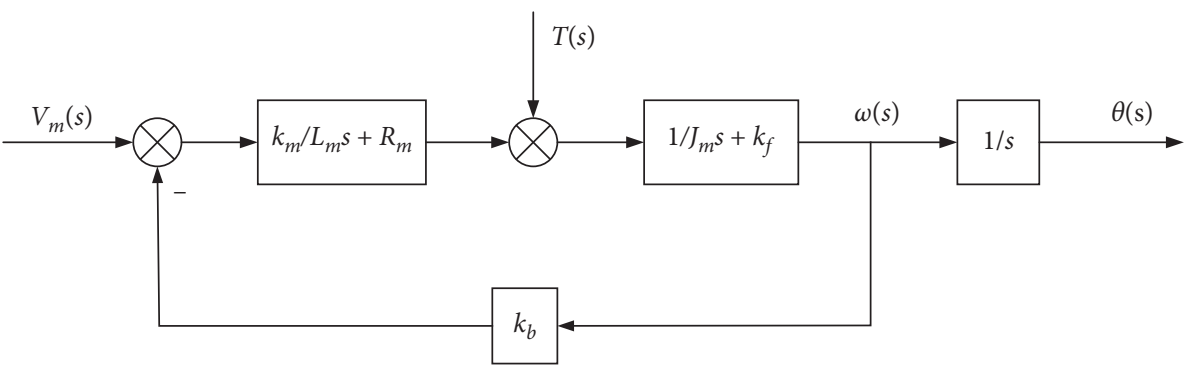

FIGURE 4: XQ WMR DC motor control structure diagram.

where $s$ is the Laplace transformation operator and $\mathscr{L}\{\cdot\}$ represents the transformation.

3.2. The Considered Fractional-Order PD Controller. Different from the traditional PD controller, there are three parameters in the proposed FOPD controller which are formulated as follows:

$$
C(s)=K_{p}+K_{d} s^{\gamma},
$$

where $0<\gamma<2$. This is a specific form of the FOPID controller which involves an integrator order $\lambda(\lambda=0$ in this paper) and a differentiator order $\gamma[36,37]$.

\section{FOPD Controller Design Specifications with Guaranteed Flat Phase Interval}

The open-loop transfer function of WMR can be achieved from the above sections as follows:

$$
G(s)=P(s) C(s)=\frac{k_{p}+k_{d} s^{\gamma}}{s(T s+1)} .
$$

Based on the definitions of gain and phase margins, the following design specifications are introduced:

4.1. Gain Crossover Frequency Specification. The amplitude of the open-loop system should be zero at its gain crossover frequency in logarithmic frequency domain. In other words, the amplitude should equal to 1 at its gain crossover frequency, which can be expressed as follows:

$$
|G(j \omega)|_{\omega=\omega_{c}}=\mid P(j \omega) C(j \omega) \|_{\omega=\omega_{c}}=1,
$$

where $\omega_{c}$ is the interested crossover frequency.
4.2. Phase Margin Specification. Denote $\varphi_{m}$ as the required phase margin, and this specification can be depicted as follows:

$$
\left.\operatorname{Arg}[G(j \omega)]\right|_{\omega=\omega_{c}}=\left.\operatorname{Arg}[P(j \omega) C(j \omega)]\right|_{\omega=\omega_{c}}=-\pi+\varphi_{m} .
$$

4.3. Flat Phase Interval Guaranteed Robustness Specification. To realize the robustness for the controlled system, the flat phase robust tuning specification can be described by the following condition at crossover frequency $\omega_{c}$ :

$$
\left.\frac{\mathrm{d}(\operatorname{Arg}[G(j \omega)])}{\mathrm{d} \omega}\right|_{\omega=\omega_{c}}=0 \text {. }
$$

However, there are a few drawbacks of the flat phase specification in equation (13), which have already been discussed in Section 1. Here, we give the following condition to improve the existing flat phase specification:

$$
\left|\frac{\mathrm{d}(\operatorname{Arg}[G(j \omega)])}{\mathrm{d} \omega}\right| \omega \in\left[\omega_{\min }, \omega_{\max }\right] \mid \leq \varepsilon
$$

where $0<\varepsilon \ll 1$ is a small enough scalar and smaller $\varepsilon$ means better flatness of the phase curve; given $\varepsilon, \omega_{\min }$, and $\omega_{\max }$ are the minimum and maximum frequency near $\omega_{c}$ $\left(\omega_{c} \in\left[\omega_{\min }, \omega_{\max }\right]\right)$ between which the flat phase interval can be guaranteed. Define $l=\omega_{\max }-\omega_{\min }$ as the width of the flat phase with regards to $\varepsilon$. Longer $l$ means better robust performance under the same $\varepsilon$. Next, we present the steps to obtain $l$.

Step 1: given $\varepsilon$, compute whether the following inequality holds or not:

$$
\left|\frac{\mathrm{d}(\operatorname{Arg}[G(j \omega)])}{\mathrm{d} \omega}\right|_{\omega=\omega_{c}} \mid<\varepsilon .
$$

If yes, go to Step 2. If not, it obtains $l=0$. 
Step 2: if inequality (3) holds, solve the next equation and gain its solutions:

$$
\left|\frac{\mathrm{d}(\operatorname{Arg}[G(j \omega)])}{\mathrm{d} \omega}\right|=\varepsilon .
$$

Let $\omega_{\min }$ and $\omega_{\max }$ be the solutions which are the nearest to $\omega_{c}$ in negative and positive directions, respectively.

Step 3: give the width $l=\omega_{\max }-\omega_{\min }$.

Remark 1. All the above steps need to be processed under a given $\varepsilon>0$. When $\varepsilon>0$ is fixed, longer $l$ could offer better robustness for the controlled system. However, an excessively small $\varepsilon>0$ may lead to $l=0$. Thus, $\varepsilon$ should be well chosen according to the practical requirements.

\section{Design Procedure}

Stability is the primary concern in control systems. So, the proposed controller design procedure is finding out the complete parameter sets which can stabilize the controlled system first, and then pick up the parameters which satisfy the proposed design specifications from the complete sets.

5.1. Control Process Stabilizing. The stabilizing process is investigated with respect to parameters of the proposed FOPD controller, namely, $k_{p}, k_{d}$, and $\gamma$.

One single closed-loop transfer function depicted in Figure 3 can be yielded according to equation (10) as follows:

$$
F(s)=\frac{G(s)}{1+G(s)}=\frac{K\left(k_{p}+k_{d} s^{\gamma}\right)}{s(T s+1)+K\left(k_{p}+k_{d} s^{\gamma}\right)} .
$$

So, the characteristic equation of the XQ WMR motor system is

$$
D\left(k_{p}, k_{d}, \gamma ; s\right)=s(T s+1)+K\left(k_{p}+k_{d} s^{\gamma}\right) .
$$

The controlled system stability is determined by the root locations of its characteristic equation in (11). If all the roots locate in the left half of $s$-domain, the system is supposed to be bounded-input bounded-output stable. Therefore, the stability region of the XQ WMR motor system can be obtained by seeking for the parameter sets which can ensure the roots of equation (11) lie in the left half of $s$-domain in $D\left(k_{p}, k_{d}, \gamma ; s\right)$. The boundaries of the stability region derived from $D\left(k_{p}, k_{d}, \gamma ; s\right)$ can be achieved by finding the corresponding IRB (infinite root boundary), CRB (complex root boundary), and RRB (real root boundary) [38]. Note that if a system is strictly proper, it will not have IRB [39]. So, only RRB and CRB are taken into consideration in this paper.

(i) RRB is defined by

$$
D\left(k_{p}, k_{d}, \gamma ; s=0\right)=K k_{p}=0 .
$$

So that $k_{p}=0$. (ii) $\mathrm{CRB}$ can be expressed as follows:

$$
\begin{aligned}
& D\left(k_{p}, k_{d}, \gamma ; s=j \omega\right) \\
& =T \omega \cos \left(\frac{\pi}{2}\right)+j T \omega \sin \left(\frac{\pi}{2}\right)+j \omega+K k_{p} \cos (\omega L) \\
& \quad-K k_{p} j \sin (\omega L)+K k_{p} \omega^{\gamma}\left[\cos \left(\frac{\pi \gamma}{2}\right)+j \sin \left(\frac{\pi \gamma}{2}\right)\right]=0,
\end{aligned}
$$

where $j^{\gamma}=e^{\gamma \pi j / 2}=\cos (\gamma \pi / 2)+j \sin (\gamma \pi / 2)$.

Here, both the real part and imaginary part of equation (20) should be equal to 0 :

$$
\begin{aligned}
& A_{1}+k_{p} K+k_{d} K \omega^{\mu} B_{1}=0, \\
& A_{2}-k_{p} K+k_{d} K \omega^{\mu} C_{1}=0,
\end{aligned}
$$

where

$$
\begin{aligned}
& A_{1}=T \omega \cos \left(\frac{\pi}{2}\right), \\
& A_{2}=T \omega \sin \left(\frac{\pi}{2}\right)+\omega, \\
& B_{1}=\cos \left(\frac{\gamma \pi}{2}\right), \\
& C_{1}=\sin \left(\frac{\gamma \pi}{2}\right) .
\end{aligned}
$$

Then, one can get from equation (21) that

$$
\begin{aligned}
& k_{p}=\frac{A_{1}-A_{2}}{K\left(B_{1}+C_{1}\right)}, \\
& k_{d}=-\frac{A_{1} C_{1}+A_{2} B_{1}}{K \omega^{\gamma}\left(C_{1}+B_{1}\right)} .
\end{aligned}
$$

Therefore, with $\omega \longrightarrow+\infty$ from 0 , the stability region of a fixed fractional order $\gamma$ composed by the corresponding $\mathrm{RRB}$ and CRB can be achieved. All parameter sets $\left(k_{p}, k_{d}\right)$ in the region can guarantee the stability of the controlled XQ WMR motor system. Then, a three-dimensional surface of $\left(k_{p}, k_{d}, \gamma\right)$ can be determined by sweeping the differential order $\gamma \in\left(0, \gamma_{\max }\right) \cdot \gamma_{\max }$ is the biggest $\gamma$ which can ensure that the control system is strictly proper. The achieved surface is the maximum stability surface of the XQ WMR system under the FOPD controller because the phase margin $\varphi_{m}$ is assumed to be 0 at this moment. Parameter sets $\left(k_{p}, k_{d}, \gamma\right)$ on this surface can ensure the stability of the controlled system; however, no design specification is satisfied at this stage.

Substitute $s$ in equations (10) and (11) by $j \omega$ as follows:

$$
\begin{gathered}
G(j \omega)=\frac{K\left(k_{p}+k_{d} \omega^{\gamma} j^{\gamma}\right)}{j \omega(T \omega j+1)}, \\
|G(j \omega)|=\frac{K\left[D^{2}+E^{2}\right]^{1 / 2}}{\left[B_{2}^{2}+C_{2}^{2}\right]^{1 / 2}}=1,
\end{gathered}
$$

where 


$$
\begin{aligned}
& D=k_{p}+k_{d} \omega^{\gamma} \cos \left(\frac{\gamma \pi}{2}\right), \\
& E=k_{d} \omega^{\gamma} \sin \left(\frac{\gamma \pi}{2}\right) \\
& B_{2}=T \omega \cos \left(\frac{\pi}{2}\right)+\omega \\
& C_{2}=T \omega \sin \left(\frac{\pi}{2}\right) .
\end{aligned}
$$

Then, one can get

$$
D^{2}+E^{2}=\frac{\left(B_{2}^{2}+C_{2}^{2}\right)}{K^{2}}
$$

Multiply both sides of equation (16) with $e^{\pi j / 2}$ and yields,

$$
\begin{aligned}
e^{j(\pi / 2)} G(j \omega) & =\frac{K\left(k_{p}+k_{d} \omega^{\gamma} j^{\gamma}\right)}{T \omega j+\omega} \\
& =K \frac{D B_{2}+E C_{2}+j\left(E B_{2}-D C_{2}\right)}{B_{2}^{2}+C_{2}^{2}} .
\end{aligned}
$$

Hence, equation (13) is obtained as follows:

$$
\operatorname{Arg}[G(j \omega)]=\arctan \left(\frac{E B_{2}-D C_{2}}{D B_{2}+E C_{2}}\right)-\frac{\pi}{2}+n \pi=-\pi+\varphi_{m}
$$

Denote

$$
N=\frac{E B_{2}-D C_{2}}{D B_{2}+E C_{2}}=\tan \left(-\pi+\varphi_{m}+\frac{\pi}{2}-n \pi\right) .
$$

From equation (28), one can obtain

$$
D=\frac{B_{2}-N C_{2}}{N B_{2}-C_{2}} E .
$$

From equations (26) and (30), E can be obtained as follows:

$$
E=\left\{\frac{B_{2}^{2}+C_{2}^{2} / K^{2}}{\left[1+\left(B_{2}-N C_{2} / N B_{2}-C_{2}\right)^{2}\right]}\right\}^{1 / 2} .
$$

Substituting $E$ in equation (26) by equation (30), $k_{p}$ and $k_{i}$ are achieved as follows:

$$
\begin{aligned}
& k_{d}=\frac{E}{\omega^{\gamma} \sin (\pi \gamma / 2)}, \\
& k_{p}=\frac{B_{2}-N C_{2}}{N B_{2}-C_{2}} E-k_{d} \omega^{\gamma} \cos \left(\frac{\pi \gamma}{2}\right) .
\end{aligned}
$$

When $\varphi_{m}$ is predefined, with $\omega \longrightarrow+\infty$ from 0 and $\gamma \longrightarrow \gamma_{\max }$ from 0 , an available stability surface can be obtained from equation (32). Then, if an interested $\omega_{c}$ is chosen, an available stability curve can be achieved. At this stage, the design specifications in equations (11) and (12) are fulfilled by parameters $\left(k_{p}, k_{d}, \gamma\right)$ on the available stability curve. Remark that $\omega_{c}$ cannot be bigger than $\omega_{m}$, where $\omega_{m}$ is determined by the intersection points of RRB and CRB. It is the maximum $\omega$ value which can ensure the stability of the control system.

From equations (14) and (28), one can obtain

$$
\begin{aligned}
& \frac{\mathrm{dArg}[G(j \omega)]}{\mathrm{d} \omega}, \\
& =\frac{\left[(D \mathrm{~d} E / \mathrm{d} \omega-E \mathrm{~d} D / \mathrm{d} \omega)\left(C_{2}^{2}+B_{2}^{2}\right)+\left(C_{2} \mathrm{~d} B_{2} / \mathrm{d} \omega-B_{2} \mathrm{~d} C_{2} / \mathrm{d} \omega\right)\left(D^{2}+E^{2}\right)\right]}{\left[\left(D B_{2}+E C_{2}\right)^{2}+\left(E B_{2}-D C_{2}\right)^{2}\right]},
\end{aligned}
$$

where

$$
\begin{aligned}
\mathrm{d} D / \mathrm{d} \omega & =\gamma \omega^{\gamma-1} k_{d} \cos \left(\frac{\pi \gamma}{2}\right), \\
\mathrm{d} E / \mathrm{d} \omega & =\gamma \omega^{\gamma-1} k_{d} \sin \left(\frac{\pi \gamma}{2}\right), \\
\mathrm{d} B_{2} / \mathrm{d} \omega & =\omega T \cos \left(\frac{\pi}{2}\right)+1, \\
\mathrm{~d} C_{2} / \mathrm{d} \omega & =\omega T \sin \left(\frac{\pi}{2}\right) .
\end{aligned}
$$

At last, the proposed FOPD parameters $\left(k_{p}, k_{d}, \gamma\right)$ are determined by checking all the parameter sets on the obtained available stability curve and finding out the one which satisfy the robust design specification in equation (14). So, the obtained FOPD controller satisfies all the three design specifications in Section 3 and can also ensure the stability of the closed-loop system.

On a whole, the design procedure can be summarized as follows:

Step 1: find the maximum stability surface of XQ WMR under the FOPD controller with $\omega \longrightarrow+\infty$ from 0 by sweeping $\gamma \in\left(0, \gamma_{\max }\right)$. The parameter sets on maximum stability surface can only guarantee the stability of the controlled system.

Step 2: define phase margin $\varphi_{m}$ and find the available stability surface. Then, choose the interested crossover frequency $\omega_{c}$, so the available stability curve can be obtained on the available stability surface. The parameter sets on available stability curve satisfy both the gain crossover frequency and phase margin specifications. 
Step 3: check all the parameter sets on the achieved available stability curve and find the one fulfill the robust design specification mostly.

\section{Experiment and Discussion}

6.1. Implementation of FOPD Controller. The implementation of the FOPD controller is one of the critical problems to achieve desirable trajectory tracking performance. The key point of the FOPD controller implementation concentrates on the approximation of the fractional operator in equation (9). In this section, the widely used Oustaloup et al. approximation method is applied without loss of generality [40].

A fractional operator can be expressed as follows:

$$
F_{o}(s)=\left(\frac{s}{w_{j}}\right)^{\beta}, \quad \beta \in R^{+} .
$$

Consider the interested approximation frequency interval as $\left(w_{1}, w_{2}\right)$, so the operator $s / w_{j}$ can be substituted by

$$
k_{0} \frac{1+s / w_{s}}{1+s / w_{b}},
$$

where $k_{0}=w_{s} / w_{j}=w_{j} / w_{b}, w_{s}<w_{1}, w_{b}>w_{2}$.

Then, equation (35) can be updated as follows:

$$
F_{o}(s)=k\left(\frac{1+s / w_{s}}{1+s / w_{b}}\right)^{\beta},
$$

where $k=k_{0}^{\beta}$.

The transfer function above can be transformed into a zero-pole form as follows:

$$
F_{o}(s)=\lim _{m \longrightarrow \infty} \widehat{F}_{o}(s),
$$

where

$$
\begin{aligned}
\widehat{F}_{o}(s) & =\left(\frac{w_{j}}{w_{l}}\right)^{\beta} \prod_{n=-m}^{m} \frac{1+s / w_{n}}{1+s / w_{n}^{\prime},} \\
w_{n} & =w_{s}\left(\frac{w_{b}}{w_{s}}\right)^{((1-\beta)+n+m) /(2 m+1)}, \\
w_{n}^{\prime} & =w_{s}\left(\frac{w_{b}}{w_{s}}\right)^{(1 / 2(1+\beta)+n+m) /(2 m+1)} .
\end{aligned}
$$

The 7th Oustaloup approximation with expected frequency range $\left(10^{-3}, 10^{3}\right)$ is used in this section.

6.2. Controller Parameters Regulation. According to the controller design procedures in Section 4, the maximum stability surface is obtained for XQ WMR in Figure 5. So, all the parameter sets on the surface can guarantee the stability of the control system.

Set the phase margin as $\varphi=45^{\circ}$, the available stability surface can be obtained, as shown in Figure 6. It can be seen that the area of the available stability surface is inversely proportional to the chosen phase margin. Then, $\omega_{c}=1$ is set as the interested crossover frequency, so the available stability curve can be obtained on the available stability surface.
The parameters on the achieved curve satisfy the gain crossover frequency and phase margin specifications.

Finally, the parameter sets on the available stability curve are checked according to the robustness specification and the proposed FOPD controller parameters are obtained as $k_{p}=19.5332, k_{d}=6.4041$, and $\gamma=0.9581$. Correspondingly, for fair comparison, a PID controller which is tuned by the optimal tuning method in [41] with similar specifications is also designed here.

Figure 7 illustrates the speed regulation comparison under the FOPD controller and PID controller with step input. It is shown that the speed regulation process under the FOPD controller has smaller rising time, accommodation time, steady state error, and almost no overshoot, which outperforms the control performance under the PID controller.

The robustness and precision comparisons are demonstrated in Figure 8 with different gain variations and external disturbance at $t=5 \mathrm{~s}$. Note that the gain variations here can be regarded as load disturbance of XQ WMR. It is shown that the speed regulation process under the FOPD controller changes little with respect to different kinds of disturbance. However, there are obvious changes in the overshoot and accommodation time of the speed regulation process under the PID controller, which means the XQ WMR system controlled by the PID controller may not achieve precise and robust control performance in practice.

6.3. Experiment. In this section, the trajectory tracking experiment results of XQ WMR are shown to verify the effectiveness of the proposed FOPD controller. For fair comparison, a traditional PD controller, whose proportional and derivative parameters are the same with that of the proposed FOPD controller, is also implemented. The reference signal is depicted as follows:

$$
\left\{\begin{array}{l}
x_{r}(t)=3 \sin \left(\frac{\pi}{45 t}\right), \\
y_{r}(t)=2 \sin \left(\frac{2 \pi}{45 t}\right),
\end{array}\right.
$$

where $x_{r}(t)$ and $y_{r}(t)$ are the $x$-axis and $y$-axis reference trajectories. The sampling time is $T=1 \mathrm{~ms}$.

The comparison of XQ WMR trajectory tracking performance is shown in Figure 9. It can be seen that the tracking performances under both controllers are acceptable. However, from the zoomed in areas of Figure 9, it is observed that the tracking performance under the proposed FOPD controller is more precise and the settling time is much shorter, which is also verified by the absolute error and $x$-axis error shown in Figures 10 and 11 illustrates the left and right speed comparisons of XQ WMR, and the control signal comparisons are demonstrated in Figure 12. These comparisons show that there is smaller oscillation in the speed control process of the proposed controller compared with the traditional PD controller, though the two controllers have the same proportional and derivative parameters. 


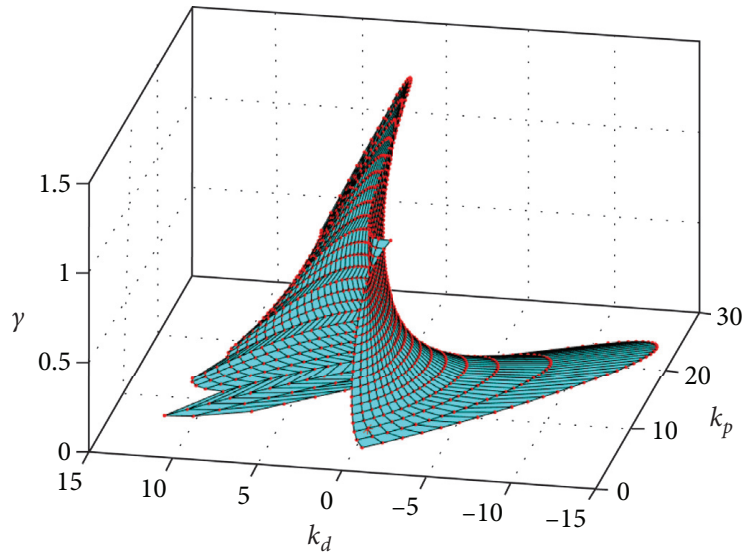

Figure 5: Maximum stability surface.

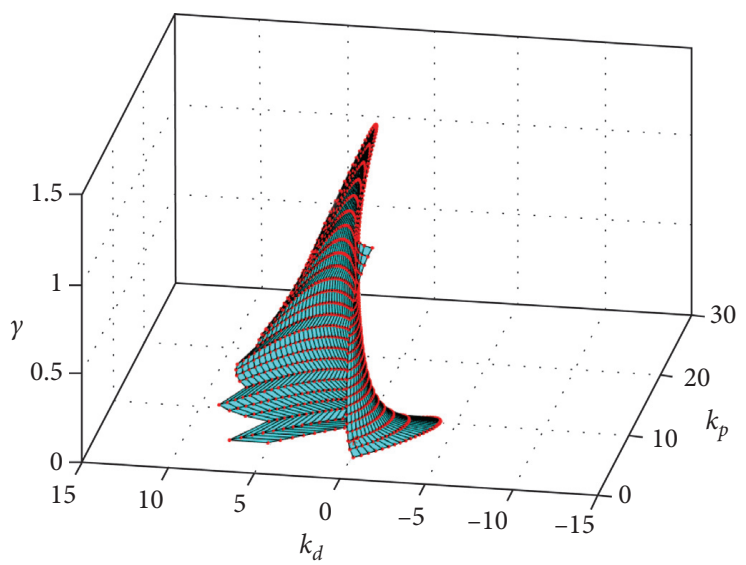

FIgURe 6: Available stability surface with $\varphi=45^{\circ}$.

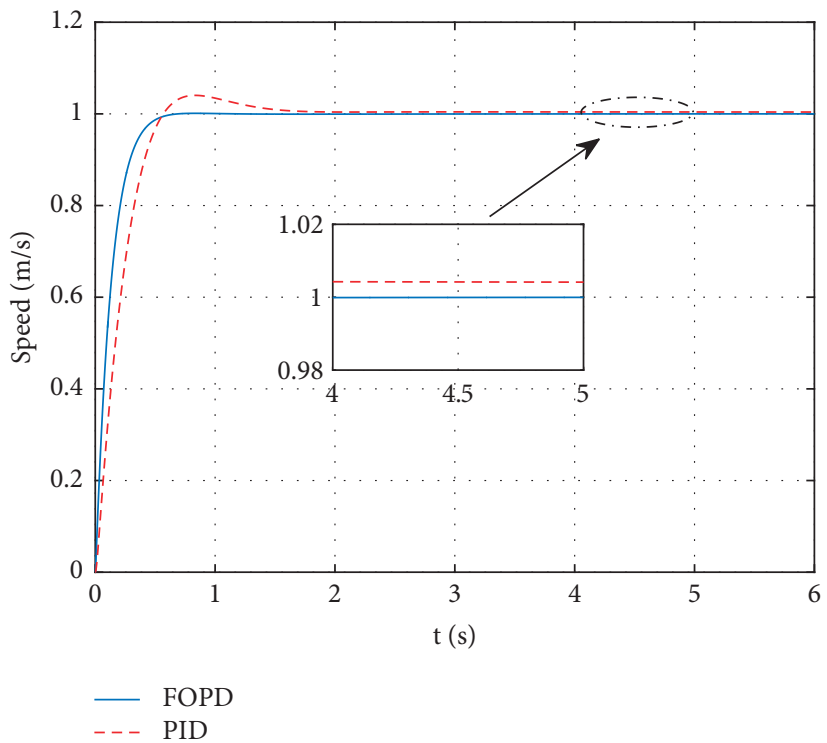

FIgURE 7: Speed regulation comparison. 

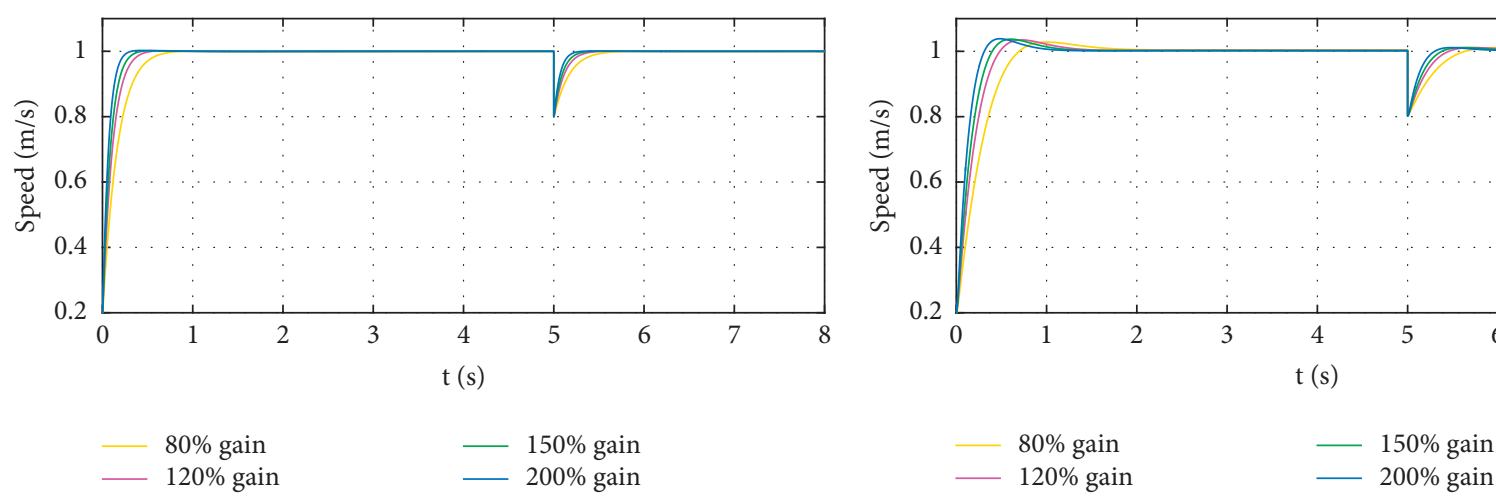

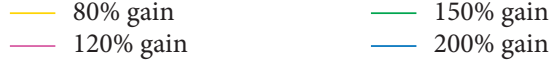

(a)

(b)

FIGURE 8: Speed regulation comparison with disturbance and gain variations: (a) FOPID and (b) PID.

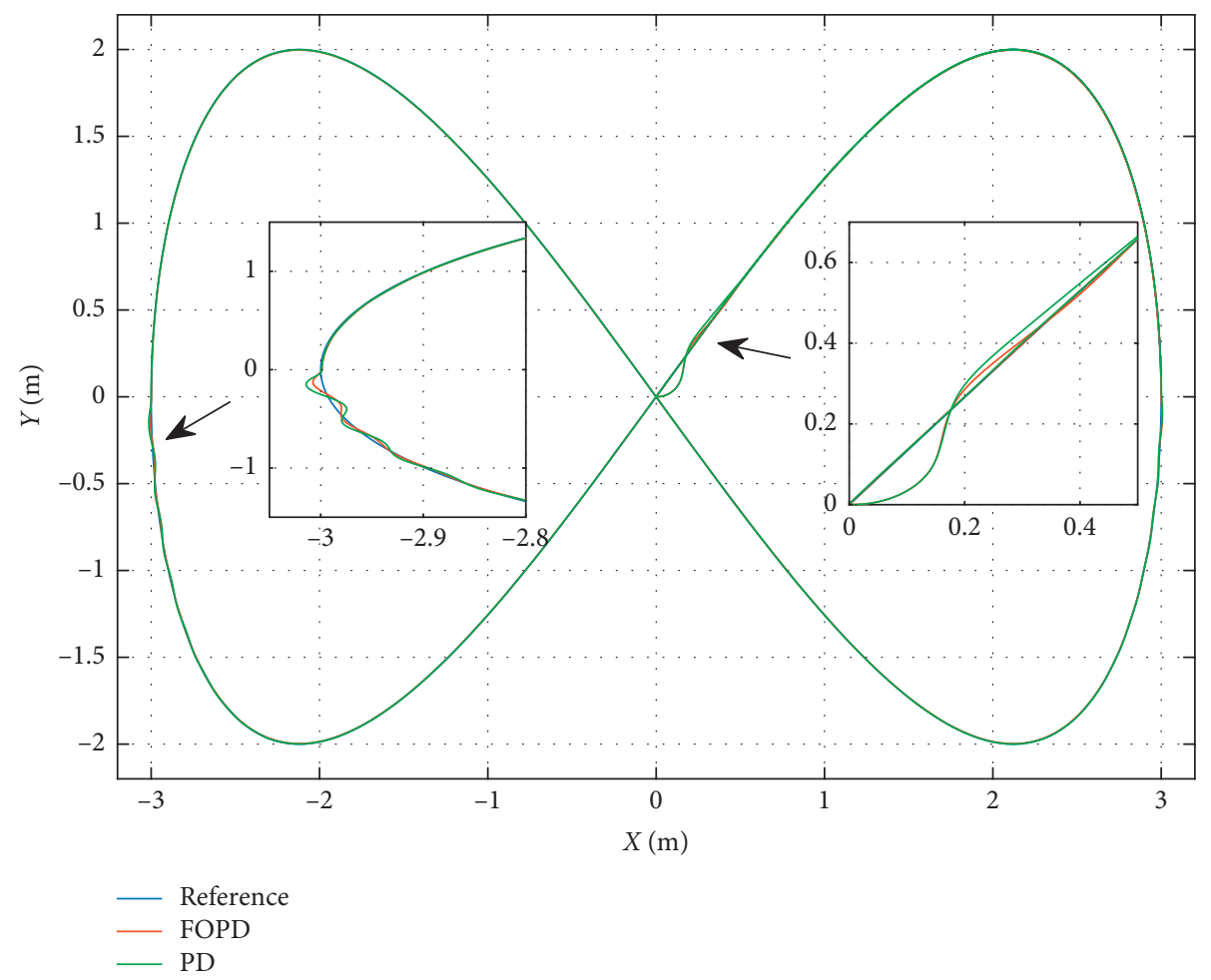

Figure 9: Trajectory tracking performance comparison.

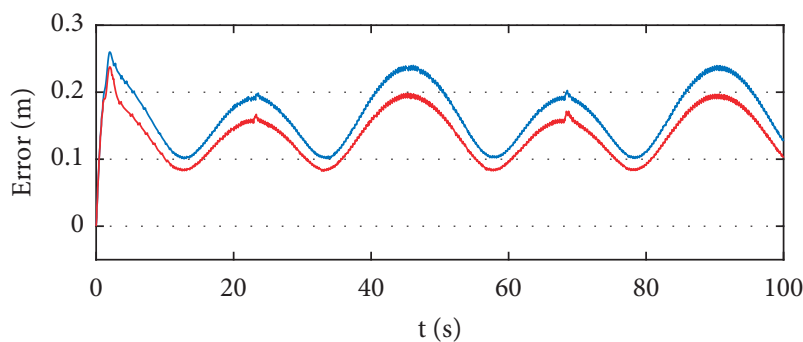

PD

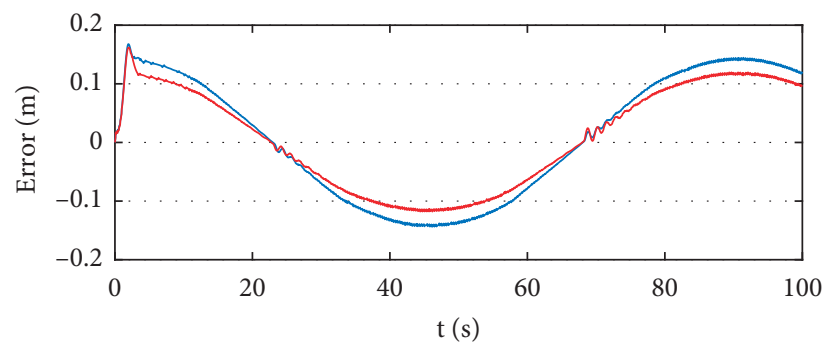

PD

— FOPD

(a)

(b)

Figure 10: Errors comparison. (a) Absolute error. (b) $\mathrm{X}$ axis error. 


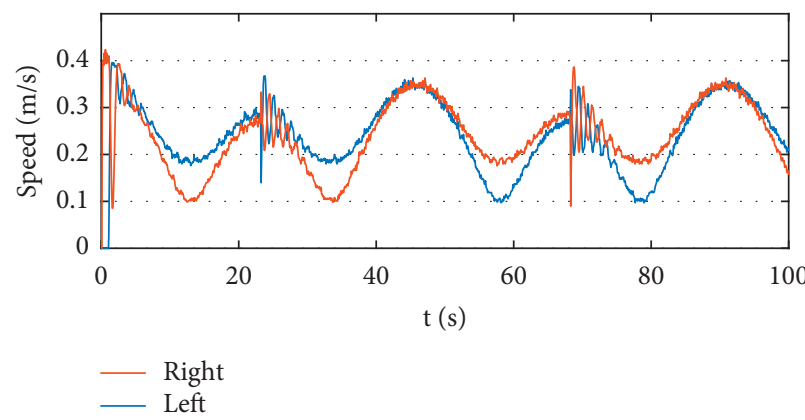

(a)

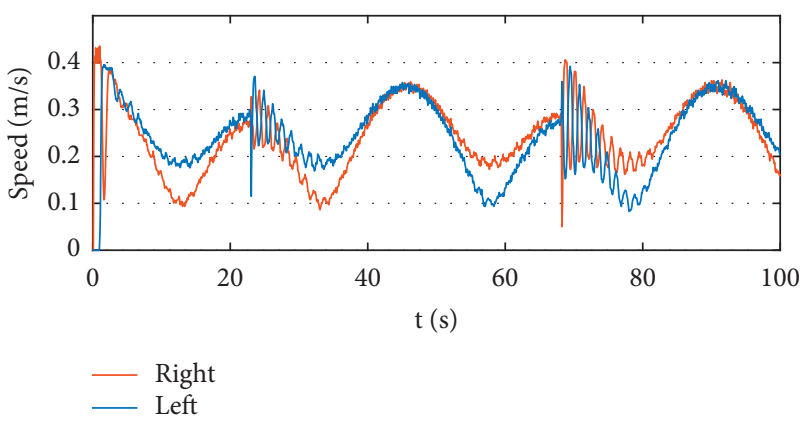

(b)

FIgURe 11: Speeds comparison. (a) FOPID and (b) PID.

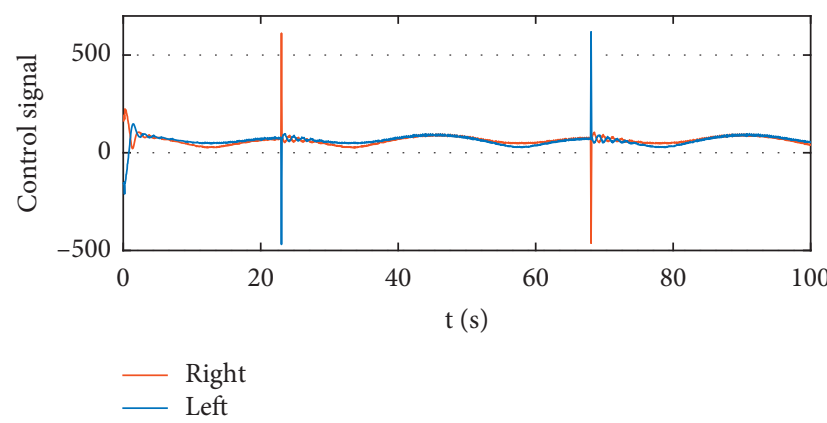

(a)

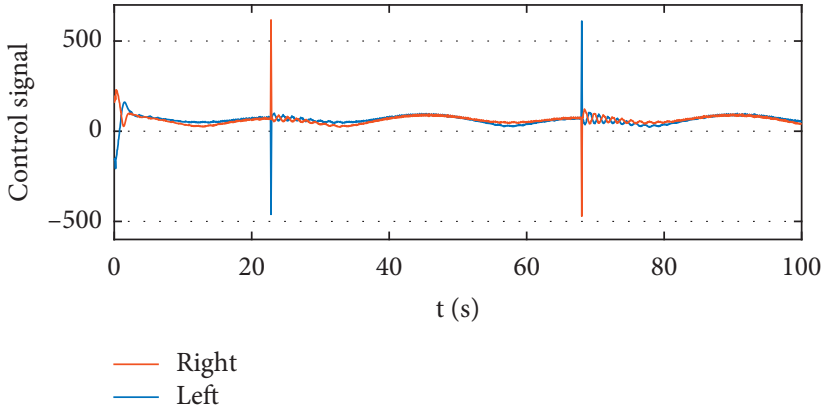

(b)

Figure 12: Control signals comparison. (a) FOPID and (b) PID.

\section{Conclusion}

In this paper, a synthesis of the FOPD controller for XQ WMR trajectory tracking control is proposed. Different from other robust FO controller tuning methods, the flat phase property can be guaranteed in a frequency interval instead of one frequency point, so the robustness of the control system is enhanced. The stabilizing process is also studied, so that the proposed FOPD controller can guarantee both the robustness and stability of the controlled system In addition, there is no complicated nonlinear equations which should be solved in this paper, so at least one parameter set which satisfies all the specifications can be found. The implementation and experiment results are presented to show the advantages of the proposed trajectory tracking algorithm. The proposed control algorithm is also capable of fulfilling different control requirements.

\section{Data Availability}

The data used to support the findings of this study are currently under embargo while the research findings are commercialized. Requests for data, 6 months after publication of this article, will be considered by the corresponding author.

\section{Conflicts of Interest}

The authors declare that they have no conflicts of interest.

\section{Acknowledgments}

This work was supported by the National Natural Science Foundation of China (Nos. 11902252 and 51979229), the Fundamental Research Funds for the Central Universities of China (Nos. G2018KY0305 and G2018KY0302), China Postdoctoral Science Foundation (No. 2019M663811), the Natural Science Foundation of Shaanxi Province (No. 2019JQ-164), and the Opening Foundation of Key Laboratory of Ocean Engineering, Shanghai Jiao Tong University (No. 1817).

\section{References}

[1] M. Chen, "Disturbance attenuation tracking control for wheeled mobile robots with skidding and slipping," IEEE Transactions on Industrial Electronics, vol. 64, no. 4, pp. 3359-3368, 2017.

[2] I. Rañó and R. Iglesias, "Application of systems identification to the implementation of motion camouflage in mobile robots," Autonomous Robots, vol. 40, no. 2, pp. 229-244, 2016.

[3] H. Peng, B. Shi, X. Wang, and C. Li, "Interval estimation and optimization for motion trajectory of overhead crane under uncertainty," Nonlinear Dynamics, vol. 96, no. 2, pp. 16931715, 2019.

[4] W. He, Y. Dong, and C. Sun, "Adaptive neural impedance control of a robotic manipulator with input saturation," IEEE Transactions on Systems, Man, and Cybernetics: Systems, vol. 46, no. 3, pp. 334-344, 2016. 
[5] H. Peng, F. Li, J. Liu et al., "A symplectic instantaneous optimal control for robot trajectory tracking with differentialalgebraic equation models," IEEE Transactions on Industrial Electronics, p. 1, 2019.

[6] X. Wang, J. Liu, Y. Zhang et al., "A unified symplectic pseudospectral method for motion planning and tracking control of 3D underactuated overhead cranes," International Journal of Robust and Nonlinear Control, vol. 29, no. 2, pp. 2236-2253, 2019.

[7] B. Li, Y. Fang, G. Hu, and X. Zhang, "Model-free unified tracking and regulation visual servoing of wheeled mobile robots," IEEE Transactions on Control Systems Technology, vol. 24, no. 4, pp. 1328-1339, 2016.

[8] C. S. Shijin and K. Udayakumar, "Speed control of wheeled mobile robots using PID with dynamic and kinematic modelling," in Proceedings of the International Conference On Innovations in Information, Embedded and Communication Systems, pp. 1-7, Coimbatore, India, March 2017.

[9] S. Zhang and L. Liu, "Normalized robust FOPID controller regulation based on small gain theorem," Complexity, vol. 2018, Article ID 5690630, 10 pages, 2018.

[10] I. Podlubny, "Fractional-order systems and PI/sup/spl lambda//D/sup/spl mu//-controllers," IEEE Transactions on Automatic Control, vol. 44, no. 1, pp. 208-214, 1999.

[11] L. Liu and S. Zhang, "Robust fractional-order PID controller tuning based on Bode's optimal loop shaping," Complexity, vol. 2018, Article ID 6570560, 14 pages, 2018.

[12] B. M. Vinagre, I. Podlubny, L. Dorcak, and V. Feliu, "On fractional PID controllers: a frequency domain approach," in Proceedings of IFAC Workshop on Digital Control Past, Terrassa, Spain, April 2000.

[13] C. A. Monje, B. M. Vinagre, V. Feliu, and Y. Chen, "Tuning and auto-tuning of fractional order controllers for industry applications," Control Engineering Practice, vol. 16, no. 7, pp. 798-812, 2008.

[14] G. Maione and P. Lino, "New tuning rules for fractional $\mathrm{PI}^{\lambda}$ controllers," Nonlinear Dynamics, vol. 49, no. 1-2, pp. 251-257, 2006.

[15] D. Valrio and C. J. Sda, "Tuning of fractional PID controllers with Ziegler-Nichols-type rules," Signal Processing, vol. 86, no. 10, pp. 2771-2784, 2006.

[16] J. J. Gude and E. Kahoraho, "Modified Ziegler-Nichols method for fractional PI controllers," in Proceedings Of IEEE International Conference On Emerging Technologies and Factory Automation, pp. 1-5, Bilbao, Spain, September 2010.

[17] V. Feliu, R. R. Prez, and L. S. Rodrguez, "Fractional robust control of main irrigation canals with variable dynamic parameters," Control Engineering Practice, vol. 15, no. 6, pp. 673-686, 2007.

[18] L. Liu, F. Pan, and D. Xue, "Variable-order fuzzy fractional PID controller," ISA Transactions, vol. 55, pp. 227-233, 2015.

[19] T. Vinopraba, N. Sivakumaran, and S. Narayanan, "IMC based fractional order PID controller," in Proceedings of IEEE International Conference on Industrial Technology, pp. 71-76, Auburn, AL, USA, March 2011.

[20] N. Sadati, A. Ghaffarkhah, and S. Ostadabbas, "A new neural network based FOPID controller," in Proceedings of IEEE International Conference on Networking, Sensing and Control, pp. 762-767, Sanya, China, April 2008.

[21] C. Yin, Y. Chen, and S.-m. Zhong, "Fractional-order sliding mode based extremum seeking control of a class of nonlinear systems," Automatica, vol. 50, no. 12, pp. 3173-3181, 2014.

[22] B. Jakovljevic, A. Pisano, M. R. Rapaic, and E. Usai, "On the slidingmode control of fractional order nonlinear uncertain dynamics," International Journal of Robust \& Nonlinear Control, vol. 26, no. 4, pp. 782-798, 2016.

[23] D. Xue, Fractional-order Control Systems Fundamentals and Numerical Implementations, Northeastern University, Shenyang, China, 2017.

[24] A. Tepljakov and A. Tepljakov, Applications of FractionalOrder Control, Springer International Publishing, Berlin, Germany, 2017.

[25] M. Ö. Efe, "Fractional order systems in industrial automationa survey," IEEE Transactions on Industrial Informatics, vol. 7, no. 4, pp. 582-591, 2011.

[26] O. Altet, X. Moreau, M. Moze, P. Lanusse, and A. Oustaloup, "Principles and synthesis of hydractive CRONE suspension," Nonlinear Dynamics, vol. 38, no. 1-4, pp. 435-459, 2004.

[27] N. Yousfi, P. Melchior, P. Lanusse, N. Derbel, and A. Oustaloup, "Decentralized CRONE control of nonsquare multivariable systems in path-tracking design," Nonlinear Dynamics, vol. 76, no. 1, pp. 447-457, 2014.

[28] S. Zhang, L. Liu, and X. Cui, "Robust FOPID controller design for fractional-order delay systems using positive stability region analysis," International Journal of Robust and Nonlinear Control, vol. 29, no. 15, pp. 5195-5212, 2019.

[29] L. Liu, S. Zhang, D. Xue, and Y. Q. Chen, "General robustness analysis and robust fractional-order PD controller design for fractional-order plants," IET Control Theory \& Applications, vol. 12, no. 12, pp. 1730-1736, 2018.

[30] A. Rojas-Moreno and G. Perez-Valenzuela, "Fractional order tracking control of a wheeled mobile robot," in Proceedings of the 2017 IEEE XXIV International Conference on Electronics, Electrical Engineering and Computing (INTERCON), pp. 1-4, Cusco, Peru, August 2017.

[31] A. Derdiyok, K. Orman, and A. Basci, "Speed and direction angle control of four wheel drive skid-steered mobile robot by using fractional order PI controller," Elektronika IR Elektrotechnika, vol. 22, no. 5, 2016.

[32] Y. Jin, Y.-Q. Chen, and D. Xue, "Time-constant robust analysis of a fractional order [proportional derivative] controller," IET Control Theory \& Applications, vol. 5, no. 1, pp. 164-172, 2011.

[33] S. Zhang, Y. Yu, and Q. Wang, "Stability analysis of fractionalorder Hopfield neural networks with discontinuous activation functions," Neurocomputing, vol. 171, pp. 1075-1084, 2016.

[34] S. Zhang, Y. Yu, and H. Wang, "Mittag-Leffler stability of fractional-order Hopfield neural networks," Nonlinear Analysis: Hybrid Systems, vol. 16, pp. 104-121, 2015.

[35] L. Liu, S. Tian, D. Xue, T. Zhang, and Y. Chen, "Continuous fractional-order zero phase error tracking control," ISA Transactions, vol. 75, pp. 226-235, 2018.

[36] H. Li, Y. Luo, and Y. Chen, "A fractional order proportional and derivative (FOPD) motion controller: tuning rule and experiments," IEEE Transactions on Control Systems Technology, vol. 18, no. 2, pp. 516-520, 2010.

[37] L. Liu, S. Zhang, D. Xue, and Y. Chen, "Robust stability analysis for fractional-order systems with time delay based on finite spectrum assignment," International Journal of Robust and Nonlinear Control, vol. 29, no. 8, pp. 2283-2295, 2019.

[38] K. Chen, R. Tang, and C. Li, "Phase-constrained fractional order PI controller for second-order-plus dead time systems," Transactions of the Institute of Measurement \& Control, vol. 39, no. 8, 2016.

[39] S. E. Hamamci, "Stabilization using fractional-order PI and PID controllers," Nonlinear Dynamics, vol. 51, no. 1-2, pp. 329-343, 2008. 
[40] A. Oustaloup, P. Melchior, P. Lanusse, O. Cois, and F. Dancla, "The CRONE toolbox for MATLAB," in An Introduction to Reservoir Simulation Using MATLAB/GNU Octave, Cambridge University Press, Cambridge, UK, 2000.

[41] L. Liu, D. Xue, and S. Zhang, "Closed-loop time response analysis of irrational fractional-order systems with numerical Laplace transform technique," Applied Mathematics and Computation, vol. 350, pp. 133-152, 2018. 